\title{
Prune belly syndrome: Approaches to its diagnosis and management
}

\author{
Radhouane Achour ${ }^{1, *}$, Wafa Bennour ${ }^{2}$, Imen Ksibi ${ }^{2}$, Meriem Cheour ${ }^{2}$, Tarek Hamila ${ }^{1}$, \\ Rim Ben Hmid ${ }^{1}$, Samia Kacem ${ }^{2}$ \\ ${ }^{1}$ Emergency Department of Gynecology and Obstetrics, Maternity and Neonatology Center, Faculty of Medicine, Tunis-El Manar \\ University, Tunis, Tunisia; \\ ${ }^{2}$ Neonatology Department, Maternity and Neonatology Center, Faculty of Medicine, Tunis-El Manar University, Tunis, Tunisia.
}

\begin{abstract}
Summary Prune Belly syndrome (PBS) or Eagle-Barrett syndrome is an anatomo-radiological syndrome consisting of a complex and rare malformation characterized by the following triad of symptoms: deficiency of the abdominal muscles, malformations of the urinary tract, and bilateral cryptorchidism. The exact etiology is unknown, though PBS predominantly occurs in males. The clinical manifestations can vary widely, from stillbirth to renal and major respiratory dysplasia to almost normal children. The current study included a total of 3 patients. The findings included clinical characteristics, diagnostics, therapy, and clinical outcomes. All patients were diagnosed with congenital aplasia of the abdominal wall and a variety of urogenital malformations. Cryptorchidism and a mega-bladder were observed in 2 patients and distinctive renal malformations, such as renal dysplasia, were observed in 1 patient. Treatment varies but usually includes surgical management of symptoms. One patient required urgent urinary surgery; a vesicotomy was urgently performed due to anuria. These aspects explain the great diversity of opinions on the approach to this syndrome, but the severity of renal dysplasia is the main prognostic factor. Two newborns died a few days later due to severe renal failure. Despite these concerns, many patients with PBS report being in physical and mental health and having a good quality of life.
\end{abstract}

Keywords: Prune belly, complex malformation, diagnosis, prognosis

\section{Introduction}

Prune belly Syndrome (PBS), also known as EagleBerrett syndrome, is an extremely rare congenital disorder ( 1 in 40,000 live births) that almost exclusively affects boys $(1,2)$. This syndrome is defined by a characteristic clinical triad consisting of abdominal muscle deficiency, severe urinary tract abnormalities, and bilateral cryptorchidism in males. The term "prunebelly" reflects the characteristic wrinkled appearance of the abdominal wall in the newborn due to the complete or partial absence of abdominal wall muscles (2).

PBS is a complex malformation disorder with wide variability in severity and clinical manifestations. Its

*Address correspondence to:

Dr. Radhouane Achour, Emergency Department of Gynecology and Obstetrics, Maternity and Neonatology Center, Faculty of Medicine, Tunis-El Manar University, Faculty of Medicine Street, Rabta 2000, Tunis, Tunisia.

E-mail: radhouane.a@live.com clinical manifestations vary from stillbirth due largely to major renal and respiratory dysplasia to an almost normal child $(2,3)$. Its etiology has yet to be determined and its prognosis depends on renal involvement $(3,4)$. Diagnosis of the syndrome should be considered during antenatal care with a thorough examination and regular prenatal follow-up.

The purpose of the current study was to describe the authors' clinical experience with children identified as having PBS and to describe the syndrome's clinical features, its diagnosis, and its treatment.

\section{Patients and Methods}

Three patients seen in the Neonatology Department of the Maternity and Neonatology Center between September 2015 and March 2016 were retrospectively examined. The following parameters were examined for each patient: age, sex, consanguinity between parents, reasons for admission, results of a physical examination, 


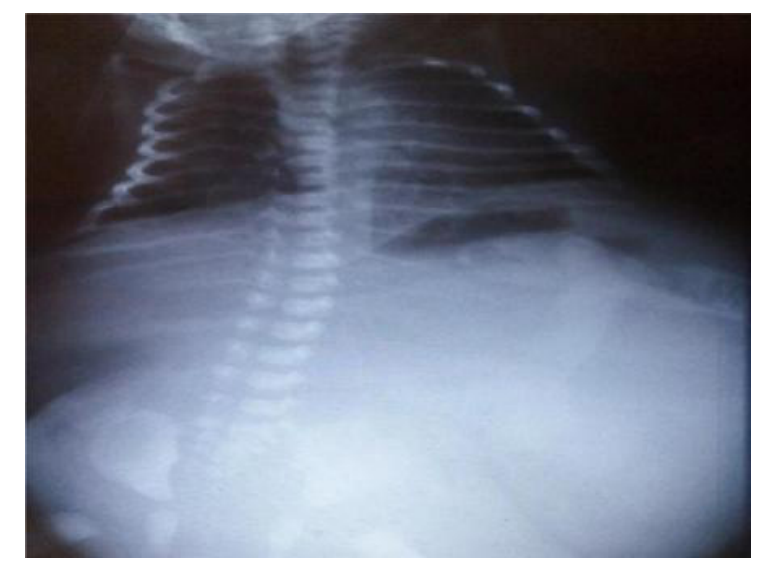

Figure 1. AXR showing a flaccid and distended abdomen in PBS.

paraclinical results (biological and radiological), and treatment (medical or surgical treatment).

\subsection{Case 1}

Baby $\mathrm{E}$ is a male born from a non-consanguineous marriage. The second twin of a bi-chorionic bi-amniotic twin pregnancy, Baby E. was delivered by caesarean section at 36 weeks due to breech presentation. There was no family history of genetic or congenital diseases. At birth, the newborn was admitted to this Department for severe bilateral uretero-pyelo-calyceal dilatation, diagnosed antenatally by ultrasound. An MRI was done at 32 weeks' gestation. The scan showed a megabladder, a small quantity of ascites, hypotonic intestinal loops, and a short femur. PBS was therefore considered. The twin was not affected.

At admission, the abdomen was bloated and hypoplasia of the abdominal musculature was evident. No abdominal masses were palpated and no obvious malformations, and especially those of the genitalia or spine, were noted. An abdominal x-ray (AXR) was performed upon admission and it showed a distended abdomen (Figure 1). An abdominal ultrasound was performed on day 2 of life, and it showed significant bladder distension associated with bilateral hydronephrosis and renal parenchymal dysplasia. Cranial sonography was normal.

The syndrome was progressively fatal. Anuria occurred on day 3 , necessitating an emergency vesicotomy. Death occurred 7 days later on day 10 of life due to severe renal failure.

\subsection{Case 2}

Baby A is a male born from a non-consanguineous marriage. The pregnancy was uncomplicated. An antenatal ultrasound was done in the fifth month of pregnancy, and it showed dilatation of the entire urinary tract with thinning of the renal parenchyma. The newborn was delivered vaginally. The newborn experienced

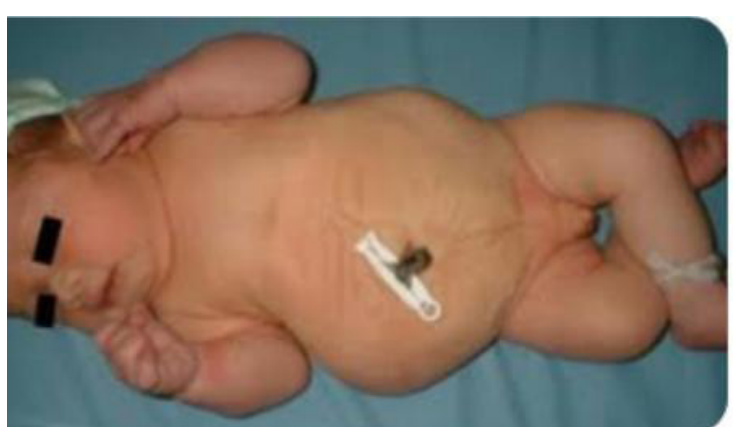

Figure 2. Hypoplasia of abdominal musculature with abdominal bloating.

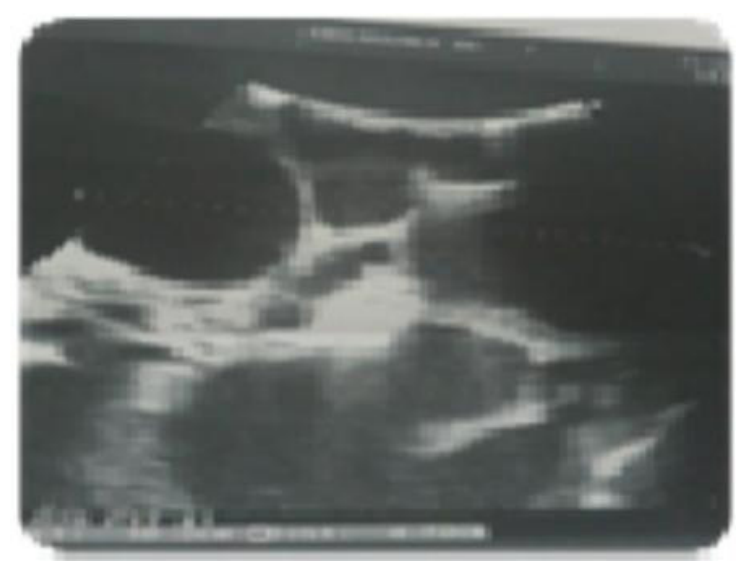

Figure 3. Significant bilateral pyelocalyceal dilatation on renal ultrasound.

respiratory distress, so it was admitted at hour 1 of life, and oxygen supplementation was required.

Upon admission, the newborn had an overdistended abdomen with hypoplasia of the abdominal musculature (Figure 2). Biological renal tests revealed abnormalities that caused severe renal failure, so an emergency abdominal ultrasound was performed. It showed significant bilateral urinary dilatation with renal parenchymal thinning and multicystic renal dysplasia (Figure 3).

Given the complexity of the urinary malformations and the poor renal prognosis, the newborn was entrusted to his parents and his chronic renal failure was managed conservatively. He died a few days later.

\subsection{Case 3}

Baby $\mathrm{M}$ is a male born from a first-degree consanguineous marriage. The newborn was delivered by emergency cesarean section at 32 weeks' gestation due to acute fetal distress and breech presentation. An antenatal ultrasound performed at 30 weeks of gestation revealed polyhydramnios, dilatation of the digestive tract suggesting a small intestinal atresia, and talipes equinovarus (clubfoot). Amniocentesis was normal. At birth, the newborn had respiratory distress. The abdomen was bloated, and the newborn had bilateral 


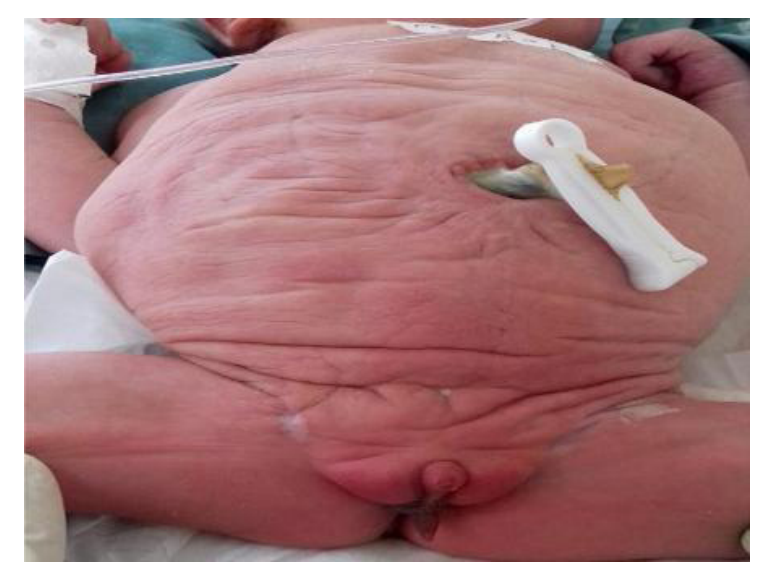

Figure 4. Abdominal bloating with bilateral cryptorchidism.

cryptorchidism and clubfoot (Figure 4).

The newborn was admitted to manage respiratory distress related to hyaline membrane disease. Continuous positive airway pressure (CPAP) was administered, and the newborn's respiration subsequently improved. An abdominal ultrasound was performed on day 2 showing a moderate dilatation of the pyelocalyceal cavities. However, renal function was normal. A point worth noting is that the fetus was diagnosed with atresia of the small intestine antenatally, but that condition was ruled out due to the passage of flatus. Thus, feeding per os was gradually introduced.

When the baby was discharged, it was referred to Pediatric Surgery. Afterwards, contact with the family was lost.

\section{Results and Discussion}

All three newborns studied were males. Consanguinity was only reported in the case of Baby M (Case 3). Moreover, Baby E had an unaffected twin, so consanguinity seemed to not be the direct cause of PBS.

In the 3 patients studied, ultrasonographic abnormalities were identified prenatally, but PBS was considered prenatally in only one case (Case 1). The syndrome was diagnosed too late into the pregnancy, precluding termination. The other two cases were diagnosed postnatally based on clinical and ultrasonographic findings.

Clinical manifestations can vary. Indeed, ectopic testes may be missed, as was true in Case 1. A skeletal abnormality (clubfoot) was noted in Case 3. Progression of the syndrome is often unpredictable. Rapid deterioration of renal function was observed in Baby $\mathrm{M}$ (Case 3); a vesicotomy was urgently performed due to anuria. This was not true in Case 2, where abdominal ultrasound showed significant bilateral urinary dilatation with thinning of the renal parenchyma and multisystem renal dysplasia. Given the complexity of the urinary malformations and the poor renal prognosis, the syndrome was treated conservatively. Both infants died a few days later due to severe renal failure.

PBS, also known as Eagle-Berrett Syndrome, is an extremely rare complex malformation with an estimated incidence of 1 in 30,000 to 50,000 births (1-4).

More than $95 \%$ of affected individuals are males $(1,3)$. In fact, fewer than 30 cases of PBS in girls have been reported in the literature $(5,6)$.

This pathology seems to be rarer nowadays as early antenatal ultrasound diagnosis is routinely performed, thus allowing termination of an undesired pregnancy (3). The etiology of PBS remains unclear. It does not seem to have a genetic basis. However, the syndrome's marked predominance in males suggests a possible genetic basis with autosomal recessive inheritance linked to sex (4). Consanguinity is not uncommon in patients with PBS, so familial factors may be involved (2).

PBS was first identified in 1950 by Eagle and Barrett (5) as a triad of congenital anomalies: deficient musculature in the anterior abdominal wall, urinary tract abnormalities, and bilateral cryptorchidism. The origin of these anomalies is not yet fully understood. Several theories have been formulated. According to one, those abnormalities are due to a prenatal obstruction of the urinary tract that leads to dilatation of the urinary tract and abdominal distension in the fetus, resulting in hypoplasia of the muscles of the abdominal wall and undescended testes $(1,5)$. According to another theory, the underlying defect in PBS is abnormal mesoderm development secondary to defective migration or differentiation of the lateral mesoderm into the abdominal musculature or muscles of the urinary tract occurring between the sixth and tenth week of gestation $(3,5)$. Aplasia of the abdominal musculature induces protrusion of the anterior abdominal wall, which gives the skin a crumpled appearance reminiscent of the skin of a "prune" and hence the name PBS.

Various urinary malformations have been observed and three clinical forms may occur (3): i) A non-viable oligoanuric form with severe renal dysplasia; ii) A severe form with significant renal dysplasia associated with megaureters, a megabladder, and progressive renal failure; and iii) A form with moderate renal dysplasia and dilatation of the urinary tract to an extent but a good prognosis.

Bilateral cryptorchidism completes the PBS triad. The causes of undescended testes are idiopathic (3). However, those clinical manifestations vary in terms of severity and when they develop. Indeed, ectopic testes may be missed in some cases along with abnormalities in the abdominal wall, which are typical of PBS $(3,5)$.

Although PBS is defined as deficient musculature in the anterior abdominal wall, urinary tract abnormalities, and bilateral cryptorchidism, it is in fact a multisystem disease, with patients displaying cardiopulmonary, gastrointestinal, and musculoskeletal anomalies to a varying degree. Indeed, several cases have been reported in the literature. According to a study by 
Routh et al. involving 133 newborns with PBS (6), pulmonary abnormalities were found in $58 \%$ of cases, and particularly pulmonary hypoplasia secondary to oligohydramnios or thoracic deformities. Cardiac abnormalities were also found in $25 \%$ of cases, as well as gastrointestinal (24\%) and skeletal abnormalities (23\%).

PBS should be diagnosed based on an antenatal ultrasound. This is of crucial importance since it would allow prompt management of newborns with the syndrome at birth, thus resulting in improved survival $(2,6)$. Indeed, PBS can be diagnosed as early as 12 weeks of gestation (6). An antenatal diagnosis should be considered whenever the following ultrasound anomalies are evident: oligohydramnios, urinary abnormalities (dilatation of the urinary tract, a megabladder, mega-ureters, and hydronephrosis), and the absence of abdominal musculature.

However, the rarity of this condition means that it is identified only in certain settings, and it is clinically diagnosed when its characteristic features are identified postnatally $(1,6)$. Patients with PBS have had a generally poor prognosis. Most patients die during the first days of life. The severity of renal dysplasia and urinary tract abnormalities and the presence of pulmonary hypoplasia are the two principal characteristics that determine the clinical manifestations of PBS and eventual outcomes for patients. Renal function can be assessed soon after birth by measuring plasma levels of urea and creatinine $(1,7)$.

The management of PBS has yet to be specifically defined because of the paucity of data. There is still debate over the appropriate treatment for PBS in early childhood. Surgical treatment includes abdominoplasty, bilateral orchiopexy, and the treatment of urinary abnormalities. Surgery is usually performed around the age of 1 year (1). In addition to its esthetic benefits, abdominal wall reconstruction helps to improve respiratory function and facilitates defecation. Moreover, it has a greater impact on the improvement of bladder emptying than that of cystoplasty $(2,8)$. Surgery is considered the only effective treatment for cryptorchidism (2). With current advances in reproductive medicine, undescended testes must be routinely repositioned to prevent infertility in patients.

The management of urinary malformations is still a subject of debate (2). Management must be tailored to each individual by striking a balance between early intervention and the undesired effects of treatments in order to improve survival and limit sequelae. The main goal of treatment should be to preserve the kidneys (3). In the event of rapid deterioration of renal function, emergency urinary diversion must be performed to allow consistent renal function, provide adequate urinary drainage, and avoid recurrent infections. Regardless of the treatment, the long-term renal prognosis is unclear. In fact, about $1 / 3$ of survivors are said to develop renal failure secondary to their renal dysplasia, obstructive nephropathy, or recurrent pyelonephritis (1). When urinary malformations are evident, the key to successful treatment is a well-trained surgeon.

In conclusion, PBS is a complex and rare malformation that is predominant in males. Renal failure is the leading cause of death (2). This syndrome is essentially a problem of treatment. The complexity of urinary malformations makes conservative treatment an important part of the therapeutic arsenal. Surgical treatment of urinary malformations must be tailored to the particular case, along with when and how that surgery is performed. These malformations should be managed by an experienced team. Orchiopexy should be performed for undescended testicles in the neonatal period to increase the chances of paternity; the same is true for abdominoplasty because of its aesthetic and functional benefits. Finally, prenatal ultrasound diagnosis is vital since it allows better management of PBS at birth.

\section{References}

1. Samal SK, Rathod S. Prune Belly syndrome: A rare case report. J Nat Sci Biol Med. 2015; 6:255-257.

2. Diao B, Diallo Y, Fall PA, Ngom G, Fall B, Ndoye AK, Fall I, Ba M, Ndoye M, Diagne BA. Prune Belly syndrome: Epidemiologic, clinic and therapeutic aspects. Prog Urol. 2008; 18:470-474. ( in French)

3. Zugor V, Schott GE, Labanaris AP. The Prune Belly syndrome: Urological aspects and long-term outcomes of a rare disease. Pediatr Rep. 2012; 4:e20.

4. Ramasamy R, Haviland M, Woodard JR, Barone JG. Patterns of inheritance in familial prune belly syndrome. Urology. 2005; 65:1227.

5. Fette A. Associated rare anomalies in prune belly syndrome: A case report. J Ped Surg Case Reports 3. 2015; 65-71.

6. Reinberg Y, Shapiro E, Manivel JC, Manley CB, Pettinato G, Gonzalez R. Prune belly syndrome in females: A triad of abdominal musculature deficiency and anomalies of the urinary and genital systems. J Pediatr. 1991; 118:395-398.

7. Routh JC, Huang L, Retik AB, Nelson CP. Contemporary epidemiology and characterization of newborn males with prune belly syndrome. Urology. 2010; 76:44-48.

8. Verdonck K, Van Eeckhout G, De Boe V, Wylock P. The prune belly syndrome: Report of a rare case and review of literature. European Journal of Plastic Surgery. 2012; 35:241-243.

(Received September 1, 2018; Revised November 5, 2018; Accepted November 21, 2018) 